cation of the circular DNA of the related Simian Virus 40 is bidirectional. Danna and Nathans have ascertained this by analysing the distribution of ${ }^{3} \mathrm{H}$-thymidine incorporated into SV40 DNA molecules replicating in vivo, making use of the Haemophilus influenzae restriction enzymes to cut the SV40 DNA at eleven specific sites to yield eleven fragments. By comparing the physical order of these fragments with the order in which they are replicated Danna and Nathans found that the fragments fall into two replication groups. Replication must therefore begin at a unique origin and proceed in two directions about the circular template, a conclusion that Slazman's group (J. Virol., 10, 484; 1972) has also arrived at recently.

\section{THERMODYNAMICS}

\section{No Carnot Dilemma?}

THE efficiency of a Carnot cycle in which the hot and cold reservoirs move at relativistic speeds is not, after all, different from the efficiency when the reservoirs are stationary. This conclusion is reached by Landsberg and Johns in a recent issue of Journal of Physics $A$ $(5,1433 ; 1972)$ and resolves a strange situation that has existed since 1934, when Tolman (Relativity, Thermodynamics and Casmology, Oxford University Press) proposed an efficiency of

$$
\eta=1-\gamma_{\mathrm{h}} T_{\mathrm{c}} / \gamma_{\mathrm{c}} T_{\mathrm{h}}
$$

(where $\mathrm{h}$ and $\mathrm{c}$ refer to the hot and cold reservoirs) rather than the conventional expression in which the relativistic factors are absent. Other investigators later produced a similar expression but with $\gamma_{0}$ and $\gamma_{h}$ interchanged. Clearly an observer in a frame of reference in which one reservoir is travelling faster than the other can "beat the Carnot efficiency" in both cases.

Landsberg and Johns have reanalysed the situation, and they pay particular attention to how much of the work done by the working fluid in one cycle is actually utilizable and should therefore be included in calculations of the efficiency. They find that one of the terms in the expression for the work done has to be replaced by another which takes account of the "purely mechanical work effectively done on the reservoirs". The efficiency they calculate then reduces to the familiar form and they conclude that motion of reservoirs and/or observer leaves the thermodynamic efficiency unchanged. Landsberg and Johns also say that the correction can be estimated by imagining a process of acceleration and one of deceleration to occur before and after the Carnot cycle respectively.

\title{
Rate of Occurrence of Supernovae
}

THE apparently high rate at which supernovae have been occurring recently in the galaxies NGC 5253 and NGC 5236 has encouraged Wamsteker et al. to take a new look at the theoretical and observational basis for the accepted supernova rate. These two galaxies are a pair which are almost certainly physically related; four supernovae have been seen in the Sc spiral NGC 5236 in the past $77 \mathrm{yr}$, and in the same period two such events have occurred in the Ir II irregular NGC 5253. This is a high frequency of supernovae compared with the average for all galaxies; the system is also interesting because the two supernovae in NGC 5253 were brighter than average, whereas the four events in NGC 5236 were fainter than average.

Although Wamsteker and his colleagues point to the Crab Nebula and its pulsar as typical of the products of supernovae, there is, of course, a school of thought which holds that the Crab is atypical. But these considerations do not affect most of the work described in next Monday's Nature Physical Science (January 1), which is concerned with the rate of supernovae to be expected in galaxies of different luminosity classes. Wamsteker et al. have made an investigation of 128 supernovae listed in van den Bergh's catalogue (Publ. David Dunlap Obs., 2, 159; 1960).

NGC 5236, for example, has an abso. lute brightness of $-20.5 \mathrm{mag}$, which places it in the brightest category; for such galaxies, the calculated supernova rate is $0.09 \mathrm{yr}^{-1}$. NGC 5253, however, has an absolute brightness of only -17.7 mag, intermediate between the third and fourth luminosity classes; the calculated frequency in this case is about 0.05 supernovae $\mathrm{yr}^{-1}$. In both cases, the prediction correlates well with the observations during the past $77 \mathrm{yr}$.

The occurrence of six supernovae in the pair within this period is thus reasonable for galaxies of these luminosities, but the curious difference in brightness between the supernovae in the two galaxies remains an enigma.

\section{Ordovician Displacements in Australia}

IN some respects, interpretations of the geology of southern Australia are not entirely satisfactory. The PrecambrianCambrian Adelaide Orogen, for example, is sigmoidal-with an added northward spur-an odd feature which has so far received no adequate explanation. Further to the south-east, in Tasmania, Precambrian rocks outcrop on the western side of the island, but there is apparently no similar outcropping only $110 \mathrm{~km}$ to the north in Victoria. Moreover, whereas Cambrian volcanism is present in Tasmania in the form of predominantly acidic (and some rather more basic) volcanics, it is represented in Victoria only by greenstones. Ordovician rocks of Tasmania and Victoria are also different, although by the Silurian palaeontological and stratigraphic histories are comparable in the two states.

In next Monday's Nature Physical Science (January 1), Crawford and Campbell show that these and other anomalies may be removed by revoking the common assumption that all "major Precambrian welded assemblages" have acted as single units during the Phanerozoic. In other words, they maintain that the traditional concept of an Australian Platform which has been stable since the end of the Precambrian can no longer be regarded as valid.

Instead, they argue, significant horizontal displacements occurred during the Ordovician, breaking up the Precambrian blocks into smaller units. The principal Precambrian blocks of Aus- tralia after the Ordovician displacements, together with the shear zones, are shown in the accompanying diagram. According to this interpretation there were two principal shears. The small Mount Painter and Willyama Blocks were probably broken off the Gawler Block at an earlier stage.

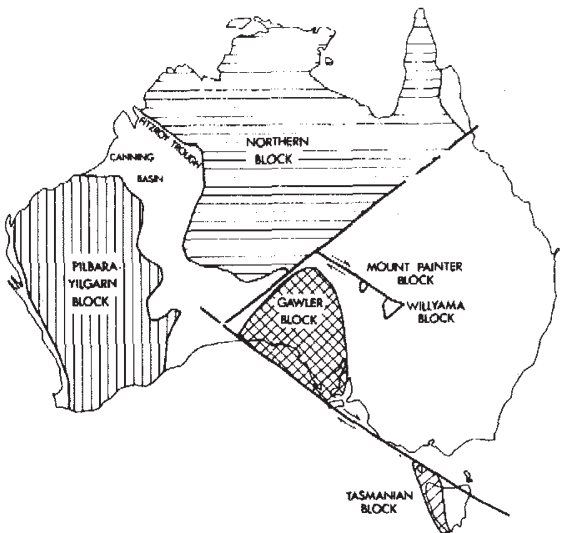

Finally, Crawford and Campbell sug gest that the cause of the Ordovician displacements may be related to the large movement of Australia-Antarctica between the Cambrian and the Devonian that is implied by palaeomagnetic data, the point being that movement of the whole continent on such a scale is unlikely to have taken place without some internal upheaval. This is an interesting thought because it implies that similar internal adjustments should also be found on other continents which have undergone largescale motion. 